\title{
Nephron-sparing surgery in multiple renal cancer: a case report
}

\begin{abstract}
BACKGROUND: In the last decade, nephron-sparing surgery has largely supplanted the radical approach for the treatment of small renal masses. More recently, ablative technologies have been discussed as alternative in patients that are not eligible for surgical approach.

CASE SUMMARY: A 54-year-old Caucasian man was referred to our Urology Clinic for multiple renal masses. A computed tomography (CT) scan revealed three contrast-enhanced lesions located in the upper pole, middle renal, and in the lower pole of the left kidney $20 \mathrm{~mm}$, $25 \mathrm{~mm}$, and $45 \mathrm{~mm}$ long, respectively. The patient underwent laparoscopic tumorectomy of two lesions and cryoablation of the left renal mass by the transperitoneal approach, performed without clamping the renal vessels. At the end of the procedure the operator posed a reno-ureteral ipsilateral stent to tutor the urinary tract. Tumor enucleation and cryoablation were chosen to preserve renal function.

CONCLUSION: This case report shows that in young patients with multiple renal tumors, cryoablation treatment is feasible and outcomes are promising as well. However, risk of complications should be considered and discussed with patients.
\end{abstract}

Keywords: Kidney Neoplasms; Cryosurgery; Nephron-sparing Surgery La chirurgia nephron-sparing nel tumore renale multiplo: un caso clinico CMI 2017; 11(1): 17-21

bttps://doi.org/10.7175/cmi.v11i1.1290

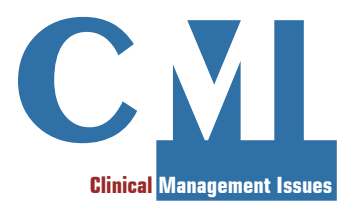

Case report
1 Urology Section, Department of Surgery, University of Catania

\section{INTRODUCTION}

Renal cell carcinoma (RCC) accounts for $2-3 \%$ of adult malignancies [1] and the incidence has increased during the last 2 decades, mostly because of the incidental detection of small, asymptomatic masses [2]. The management of localized renal cell carcinoma has evolved toward minimally invasive and nephron-sparing surgery (NSS) [3].With the increasing application of minimally invasive surgery, ablative technologies have been investigated as an alternative. They include cryoablation (CA), radiofrequency ablation (RFA), microwave, high-intensity focused ultrasound, laser interstitial thermotherapy, microwave thermotherapy, and radiosurgery [4]. Tissue is destroyed in situ, thereby avoiding the complications induced by renal ischemia and surgical excision. However, only CA and RFA have encountered a widespread use: several case series reported short-to-intermediate-term results [1]. Therefore, if surgical and oncological

\section{Why we describe this case}

Current guidelines suggest that treatment with cryoablation can be an option for the treatment of small renal masses in selected patients. Here we report the case of a 54-year-old man with multiple renal lesions successfully undergone to cryoablation in one lesion
Corresponding author Dr. Giorgio Ivan Russo giorgioivan@virgilio.it

\section{Disclosure}

The authors declare they have no competing financial interests

concerning the topics of this article 


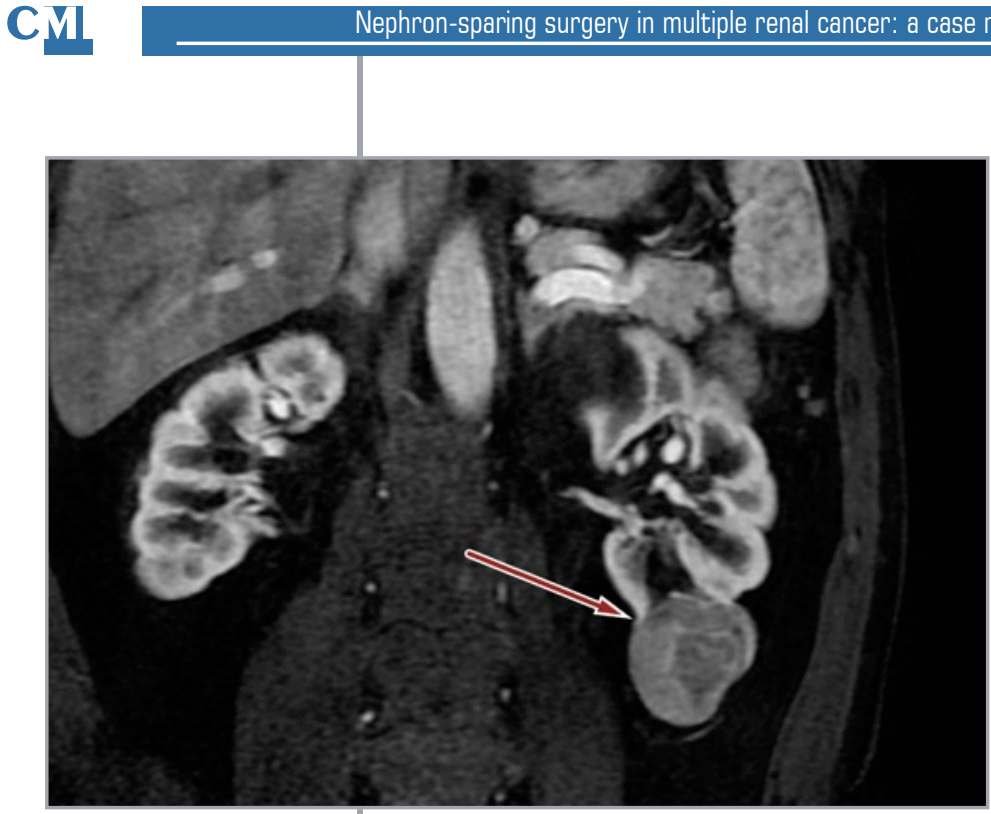

Figure 1. Intravenous contrast abdominal computed tomography showing a contrastenhancing $45 \mathrm{~mm}$ diameter solid lesion (red arrow) in the left kidney.

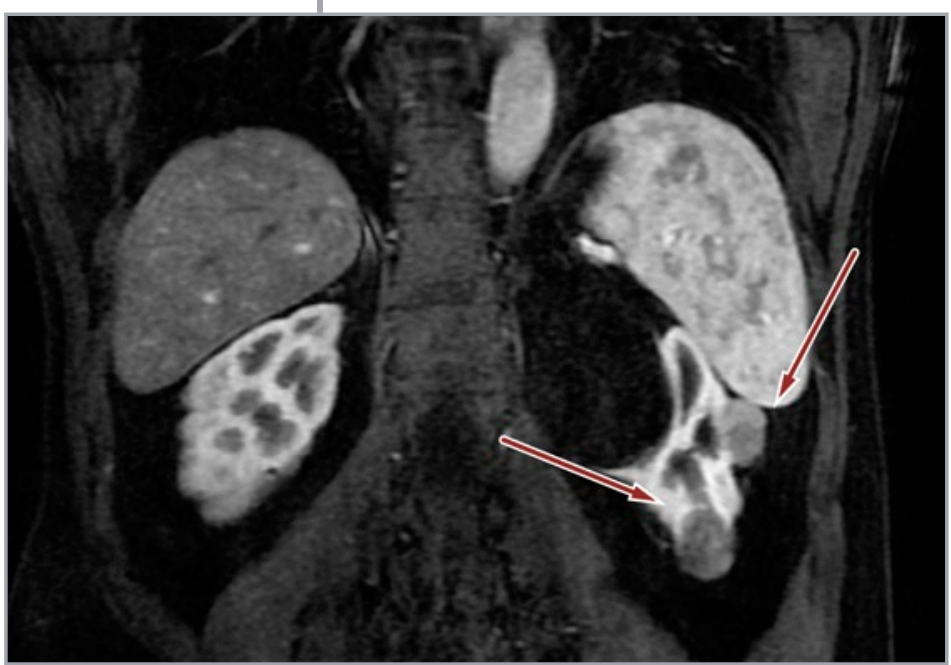

Figure 2. Intravenous contrast abdominal computed tomography showing two contrastenhancing solid lesions $20 \mathrm{~mm}$ in diameter (red arrow - the upper one) and $25 \mathrm{~mm}$ in diameter (red arrow - the lower) in the left kidney. outcomes support the feasibility and efficacy of laparoscopic cryoablation (LRC) for renal tumors, and safety can be maintained during the surgical procedure, this method may provide an alternative nephron-sparing surgery for selected patients [1]. Recently, Nielsen et al. in a large multi-institutional study reported satisfactory intermediatemasses (SRMs). Unfortunately, no randomized controlled trials comparing nephronsparing surgery and cryoablation has been undertaken.

\section{CASE REPORT}

A 54-year-old Caucasian man was admitted to our Urology Clinic due to incidentally detected renal masses on the left side. His past medical history showed hypertension and hypercholesterolemia. He had no past surgical history. His renal functions and glomerular filtration rate were normal. Contrast-Enhanced Computed Tomography (CECT) revealed multi-focal hypodense renal masses and contrast enhancement. The features of the masses were suggestive of multiple renal cell carcinoma without dissemination (Figures 1 and 2).

Tumor masses were located in the upper pole, middle renal, and in the lower pole of the kidney. Pre-operative risk in our institution was defined using PADUA and RENAL score. The lesion in the upper pole had a maximum diameter of $20 \mathrm{~mm}$ and had no communication with the urinary tract. PADUA score was $7 p$ and RENAL score was $5 \mathrm{a}$. The lesion located on the middle line had maximum diameter of $25 \mathrm{~mm}$ and was closed with the urinary tract, in fact PADUA score was $9 a$ and RENAL score was $7 a$. The lower one had maximum diameter of $45 \mathrm{~mm}$ and reached urinary tract as well. Because of the size, it had PADUA score $=9 \mathrm{a}$ and RENAL score $=10 \mathrm{a}$. According to TMN classification, the patient was classified as T2bNOM0 clinical stage. The Charlson comorbidity score was 0 .

The patient underwent laparoscopic transperitoneal approach. After dissection of the perirenal fat, macroscopically the kidney showed a prominent area at the lower pole corresponding to the lesion. Identification of other tumor masses was performed by intra-abdominal ultrasonography. The patient underwent laparoscopic renal cryoablation of the renal upper tumor and tumor enucleation of the middle renal and lower renal masses. At the end of the procedure, a double J reno-ureteral stent was posed. It was performed without clamping the renal vessels.

Postoperative laboratory examinations resulted within normal values and renal function did not change. The stent was removed 20 days after surgery. Histopathological examinations suggested a diagnosis of type 1 papillary renal cell carcinoma and clear cell renal cell carcinoma. No adjuvant therapy following surgery was recommended.

2 months after ablative treatment, the patient was hospitalized due to the occurrence of hyperpyrexia. An abdominopelvic CT scan identified a leakage closed to middle renal space. The patient was treated by systemic therapy including antibiotic therapy (grade II as Clavien-Dindo Classification 
What should the clinician ask him/herself

- Which is patient's renal function in terms of serum creatinine and glomerular filtration rate?

- How old is the patient?

- Which are renal masses locations?

- Which are the enhancement characteristics at CT scan of such lesions?

- How large are the lesions and are they close to urinary tract?

of Surgical Complications) and discharged from hospital within 12 days. The level of serum creatinine was $0.93 \mathrm{mg} / \mathrm{dl}$ immediately before treatment and $1.18 \mathrm{mg} / \mathrm{dl}$ after treatment.

At last follow up, ten months after surgery, the patient had no recurrence and renal function was stable.

\section{DISCUSSION}

NSS for patients with sporadic ipsilateral renal tumors shows excellent long-term oncological outcomes. Krambeck et al. reported their experience with multiple renal tumors treated with radical nephrectomy or NSS and found $90 \%$ and $96 \%$ cancer specific survival, respectively. Thus, in the current era of expanding indications for NSS, patients with multiple renal tumors should be considered candidates for nephron-sparing. This is particularly important, given the higher risk of subsequent metachronous lesions in the contralateral kidney in these patients $[5,6]$.

In the last decade, long-term data revealed safe oncological outcomes in patients treated for small kidney cancers with nephronsparing surgery. More recently, minimally invasive modalities for treatment of small renal masses (SRM) have been investigated as alternative to partial nephrectomy.

Although partial nephrectomy remains the gold standard, cryoablation is becoming apparent and in selected patients could be a valid alternative. Cryoablation can be performed percutaneously in the radiology suite, or laparoscopically without the need for hilar clamping. Unfortunately, only short- and intermediate-term data are available, but they seem to be promising, especially in patients who are considered poor candidates for more involved surgery. Numerous clinical trials have been published on cryoablation, often involving just a limited number of patients [7]. Ideally, ablative treatment should be compared with partial nephrectomy $(\mathrm{PN})$ in a randomized and prospective setting.
In our report, we synthetize the existing evidence to treat SRM with cryoablation. When choosing CA, renal preservation, oncological outcomes, and related complications should be considered. Thermal ablation allows preservation of renal function and studies data highlight that renal preservation is superior when compared to partial or radical nephrectomy [8]. Tumors are treated in situ without need for isolation and clamping of the renal hilum, thus contributing to preserve renal function outcomes avoiding kidney ischemia. Up to now, randomized controlled trials aimed at identifying the magnitude of impact of ablation on global renal function are still lacking.

In 2012, Wehrenberg-Klee et al. examined the impact of thermal ablation on kidney function in 48 patients with baseline chronic kidney disease (CKD). The Authors estimated that percutaneous ablative treatment (radiofrequency and CA) did not negatively impact on renal function. In fact, the mean overall renal function did not change between baseline, at 1 month and at 1 year after treatment [9].

In 2014, Thompson et al. studied oncological outcome among patient treated with $\mathrm{PN}$ and CA comparing with those treated with percutaneous radiofrequency ablation for cT1 disease [10]. They concluded that in a cohort of 1424 patients with sporadic cT1 renal masses, local recurrence-free survival (LRFS) was similar among the three treatments. Instead, metastases-free survival (MFS) was significantly better after PN and cryoablation when compared with RFA. A subgroup of 379 cT1b patients LRFS and MFS resulted similar between $\mathrm{PN}$ and cryoablation.

Atwell et al., in a sample of 115 tumors managed with percutaneous cryoablation (PCA), reported technical success without enhancement after procedure in $97 \%$ of cases and no evidence of local progression as new enhancement or growth of the ablation site in 80 tumors that were followed for a mean of 13.3 months [11]. 
More recently, in EuRECA study, Nielsen et al. have reported long-term follow up study. They investigated oncological outcomes and complication rates in patients treated with LCA between 2005 and 2015 [12]. The study has been conducted in a large cohort and the Authors concluded that LCA demonstrates satisfactory long-term oncological outcomes for T1a: in fact, the $5-/ 10$-year disease free survival (DFS) was $90.4 \% / 80.0 \%$ and $5-/ 10$-year overall survival (OS) was $83.2 \% / 64.4 \%$, respectively. The secondary aim of the study was to evaluate the occurrence of postoperative complications within 30 days of treatment. Postoperative complications were defined according to the Clavien-Dindo classification system [13]. Any complication having grade $\geq$ IIIa was defined as severe. They reported rate of postoperative complication of $16.6 \%$, with severe complications (grade $\geq$ III) of $3.2 \%$.

Therefore, although LCA is considered a minimally invasive technique, risk of complications should be considered. The most common complications found in literature are hemorrhage, perinephric hematoma, and urine leak. Other complications reported are flank pain, perinephric hematoma, and cardiovascular complications, including arrhythmia, deep venous thrombosis, hypoand hypertension and cases of myocardial infarction.

Kapoor et al. in their review reported rate of complications of $13 \%$ when evaluated using the Clavien-Dindo system and $7 \%$ when using the Common Terminology Criteria for Adverse Events grading system [14]. Sprenkle et al. reported complications rate of $13 \%$ approximately as well [15]. Sidana et al., in a sample of 162 patients undergone to PCA, LCA, or open CA, reported complication rates higher, up to $23.5 \%$ (complications were categorized using the Clavien-Dindo system). No independent risk factors, such as tumor size or the number of cryoprobes used were noted to be associated with increased risk for perinephric hematoma formation [16].

\section{CONCLUSIONS}

Thermal ablation is an increasingly used treatment option in the management of SRMs. The choice should be considered in reason of age, preoperative renal insufficiency, solitary kidneys or multiple renal lesions balancing with the risk of preoperative complications. However, due to the limited experience on cryoablation, larger studies including the long-term outcome are needed and prospective studies are requisite the better to define the role of ablative therapy in the treatment of small kidney tumors.

\section{ACKNOWLEDGEMENTS}

Written informed consent was obtained from the patient for the publication of the present case report and accompanying image.

Key points

- The management of localized renal cell carcinoma has evolved toward minimally invasive and nephron-sparing surgery, including cryoablation

- Although partial nephrectomy remains the gold standard, cryoablation is becoming apparent and in selected patients could be a valid alternative

- In particular, cryoablation can be performed percutaneously in the radiology suite, or laparoscopically without the need for hilar clamping, thus allowing preservation of renal function and avoiding the risk for kidney ischemia

- However, to date, only short-and intermediate-term data are available: larger studies including the long-term outcome are needed the better to define the role of ablative therapy in the treatment of small kidney tumors 


\section{REFERENCES}

1. Ljungberg B, Bensalah K, Canfield S, et al. EAU guidelines on renal cell carcinoma: 2014 update. Eur Urol 2015; 67: 913-24; https://doi.org/10.1016/j.eururo.2015.01.005

2. Pantuck AJ, Zisman A, Belldegrun AS. The changing natural history of renal cell carcinoma. J Urol 2001; 166: 1611-23; https://doi.org/10.1016/S0022-5347(05)65640-6; https://doi. org/10.1097/00005392-200111000-00003

3. Hafez KS, Novick AC, Butler BP. Management of small solitary unilateral renal cell carcinomas: impact of central versus peripheral tumor location. J Urol 1998; 159: 1156-60; https://doi. org/10.1097/00005392-199804000-00010; https://doi.org/10.1016/S0022-5347(01)63537-7

4. Seror O. Ablative therapies: Advantages and disadvantages of radiofrequency, cryotherapy, microwave and electroporation methods, or how to choose the right method for an individual patient? Diagn Interv Imaging 2015; 96: 617-24; https://doi.org/10.1016/j.diii.2015.04.007

5. Abreu AL, Berger AK, Aron M, et al. Minimally invasive partial nephrectomy for single versus multiple renal tumors. J Urol 2013; 189: 462-7; https://doi.org/10.1016/j.juro.2012.09.039

6. Klatte T, Patard JJ, Wunderlich $\mathrm{H}$, et al. Metachronous bilateral renal cell carcinoma: risk assessment, prognosis and relevance of the primary-free interval. J Urol 2007; 177: 2081-6; discussion 6-7; https://doi.org/10.1016/j.juro.2007.01.122

7. Gervais DA, McGovern FJ, Wood BJ, et al. Radio-frequency ablation of renal cell carcinoma: early clinical experience. Radiology 2000; 217: 665-72; https://doi.org/10.1148/ radiology.217.3.r00dc39665

8. Raman JD, Jafri SM, Qi D. Kidney function outcomes following thermal ablation of small renal masses. World J Nephrol 2016; 5: 283-7; https://doi.org/10.5527/wjn.v5.i3.283

9. Wehrenberg-Klee E, Clark TW, Malkowicz SB, et al. Impact on renal function of percutaneous thermal ablation of renal masses in patients with preexisting chronic kidney disease.J Vasc Interv Radiol 2012; 23: 41-5; https://doi.org/10.1016/j.jvir.2011.09.002

10. Thompson RH, Atwell T, Schmit G, et al. Comparison of partial nephrectomy and percutaneous ablation for cT1 renal masses. Eur Urol 2015; 67: 252-9; https://doi.org/10.1016/j. eururo.2014.07.021

11. Atwell TD, Vlaminck JJ, Boorjian SA, et al. Percutaneous cryoablation of stage T1b renal cell carcinoma: technique considerations, safety, and local tumor control. J Vasc Interv Radiol 2015; 26: 792-9; https://doi.org/10.1016/j.jvir.2015.02.010

12. Nielsen TK, Lagerveld BW, Keeley F, et al. Oncological outcomes and complication rates after laparoscopic-assisted cryoablation: a European Registry for Renal Cryoablation (EuRECA) multi-institutional study. BJU Int 2017; 119: 390-5; http://dx.doi.org/10.1111/bju.13615

13. Dindo D, Demartines N, Clavien PA. Classification of surgical complications: a new proposal with evaluation in a cohort of 6336 patients and results of a survey. Ann Surg 2004; 240: 20513; https://doi.org/10.1097/01.sla.0000133083.54934.ae

14. Kapoor A, Touma NJ,Dib RE. Review of the efficacy and safety of cryoablation for the treatment of small renal masses. Can Urol Assoc J 2013; 7: E38-E44; https://doi.org/10.5489/cuaj.193

15. Sprenkle PC, Power N, Ghoneim T, et al. Comparison of open and minimally invasive partial nephrectomy for renal tumors 4-7 centimeters. Eur Urol 2012; 61: 593-9; https://doi. org/10.1016/j.eururo.2011.11.040

16. Sidana A, Aggarwal P, Feng Z, et al. Complications of renal cryoablation: a single center experience. J Urol 2010; 184: 42-7; https://doi.org/10.1016/j.juro.2010.03.013 